\title{
Cardiopulmonary exercise testing for the selection of patients undergoing surgery for lung cancer: friend or foe?
}

\author{
Eric Lim, ${ }^{1}$ Michael Beckles, ${ }^{2}$ Chris Warburton, ${ }^{3}$ \\ David Baldwin ${ }^{4}$
}

\begin{abstract}
The contribution of exercise testing for risk assessment for lung resection is well established and has been embedded in international guidelines from Europe ${ }^{1}$ and the USA. ${ }^{2}$ There are many forms of exercise tests ( 6 min walk, 12 min walk, shuttle walk, stair climbing), but the most established investigation is formal assessment of maximum oxygen consumption during exercise $\left(\mathrm{VO}_{2}\right.$ max). British and American (American College of Chest Physicians (ACCP)) guidelines use $\mathrm{VO}_{2}$ max as the ultimate assessment of operative risk, positioned at or near the end of the functional algorithm, ${ }^{3}$ whereas European guidelines recommend the use of this test much earlier in patients with a forced expiratory volume in $1 \mathrm{~s}\left(\mathrm{FEV}_{1}\right)$ or carbon monoxide transfer factor (Tlco) $<80 \%$ predicted. ${ }^{12}$
\end{abstract}

Numerous cohort studies and a metaanalysis report the association of low $\mathrm{VO}_{2}$ max and 'high risk' lung resection. ${ }^{4-18}$ However 'high' is not quantified and 'risk' is not defined, two fundamentally important definitions if guidelines that use these terms are to be applied clinically. Here we focus on validity of the $\mathrm{VO}_{2}$ max studies and the clinical utility of the available evidence with respect to individual interpretation of risk.

\section{SAMPLE SIZE AND PRECISION OF RISK ESTIMATION OF DEATH}

Arguably, the most important outcome when considering surgery for lung cancer

${ }^{1}$ Imperial College and Academic Division of Thoracic Surgery, The Royal Brompton Hospital, London, UK; ${ }^{2}$ Department of Acute Medicine, The Royal Free Hospital, London, UK; ${ }^{3}$ Department of Respiratory Medicine, Aintree Chest Centre, Liverpool, UK; ${ }^{4}$ Respiratory Medicine Unit, David Evans Research Centre, Nottingham University Hospitals, Nottingham, UK

Correspondence to Eric Lim, Academic Division of Thoracic Surgery, Royal Brompton Hospital, Sydney Street, London SW3 6NP, UK; e.lim@rbht.nhs.uk is the ability to survive the procedure. The most apparent limitation of the currently available evidence is the lack of appropriately powered studies to address this. The precision of a risk model is not specifically dependent on sample size, but rather the number of events-that is deaths-an uncommon outcome in thoracic surgery. In the UK, lobectomy, the most common procedure for lung cancer, carried an operative mortality of $\sim 2 \%$ in $2004-2005,{ }^{19}$ and in the USA the mortality rate has been reported to range from $2.3 \%$ to $4.1 \%{ }^{20}$ Reflective of this, the largest study in this context on $\mathrm{VO}_{2}$ max (422 patients) had only 15 deaths. What is clearly more disconcerting is that publications for which recommendations on estimation of operative mortality risk have been based have sample sizes ranging from 8 to $160 .^{21}$

\section{UPPER LIMITS OF UNCERTAINTY FOR SAFE CUT-OFF VALUES}

Many studies have defined arbitrary cut-off values ranging from 15 to $20 / \mathrm{ml} / \mathrm{kg} / \mathrm{min}$ as a 'safe' cut-off value ${ }^{4-14}$ because above these levels no patient experienced an adverse event. What is the validity of this type of recommendation?

The answer lies in the uncertainly that surrounds the observation of no events (ie, upper $95 \% \mathrm{CI}$ ), a function of the sample size. For a standard binomial distribution, the upper limit of the CI of zero events with the sample size of 8-160 corresponds to $42-2.7 \%$, respectively (figure 1 ), illustrating high limits of uncertainty in the majority of studies with smaller sample sizes.

\section{ALTERNATIVE MODELS FOR RISK ESTIMATION OF DEATH}

Given these limitations, are there any other alternatives for the risk assessment for operative morality? Thoracoscore is a composite scoxring system that can be used to quantify risk. It is a logistic regression-derived model with coefficients provided for individual risk factors, calculated to provide a percentage probability of death. It is currently the best model and was developed from a sample size of 15183 patients with 338 deaths, and provides excellent discrimination with an area under the curve of $0.82 .^{22}$ Furthermore, it has been validated in different populations. ${ }^{23}$ Apart from superior statistical power, much larger sample size, external validity and excellent performance, the logistic risk model carries two further attractive advantages compared with $\mathrm{VO}_{2}$ max assessment: it is cost free and can be universally available.

\section{OTHER OUTCOMES AND COMPOSITE END POINTS}

The consistent message that lower values of $\mathrm{Vo}_{2}$ max are associated with higher risk of complications is to be expected as a measure of cardiovascular fitness. From a patient's and clinician's perspective, however, the nature of the complications is of central importance. All studies to date have used composite end points and, when multiple outcomes are combined, it becomes difficult to interpret the impact of each individual component. It has been recommended that each outcome should have a similar weighting or clinical importance to facilitate clinical interpretation. ${ }^{24}$ For example, death and myocardial infarction would be combined to estimate the total number of patients that may have experienced a myocardial infarction and survived, added to those that have (presumably) experienced a myocardial infarction and died.

Researchers, however, may use composite outcomes to increase the power of the study (by increasing event rate) and therefore increasing the chances of achieving statistically significant results. ${ }^{25}$ The corollary is that important outcomes such as death can be piggybacked within the pool of less important outcomes such as atelectasis ${ }^{5}$ 7-9 $11-1416$ or purulent sputum, ${ }^{4}$ giving rise to considerable difficulties for the clinician and, more importantly, the patient to evaluate the importance of the overall result. We believe that most patients would not consider readmission to the intensive care unit $^{11}$ atelectasis, ${ }^{5}$ 7-9 $11-14 \quad 16$ arrhythmia $^{4}$ 6-8 101314 or postoperative $\mathrm{CO}_{2}$ retention ${ }^{6} 8-101316$ as 'prohibitive' complications leading them to refuse surgery. 


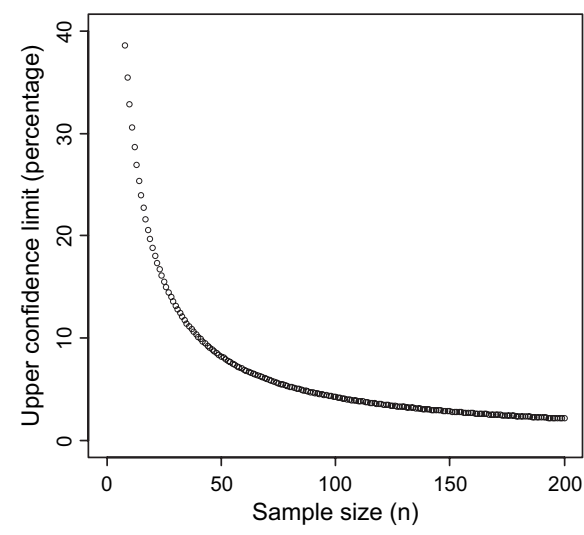

Figure 1 Upper binomial confidence limit for 'no observations'.

\section{QUANTIFICATION AND INTERPRETATION OF RISK}

A clear explanation of risks and benefits is central to good consenting practice when offering treatment options to our patients. Dichotomous categorisation of 'high' and 'standard' risk using $\mathrm{VO}_{2}$ max for risk assessment, accompanied by a combination of varied outcomes (some of which have little influence on patient decision making) renders the information difficult to apply in practice. The lack of a numerical estimate leads to subjective interpretation of 'high'; moreover, many studies do not document the uncertainty (confidence limits) that surround their estimates. As there is no accepted level of baseline risk, it is not possible to quantify the relative magnitude of 'high' to facilitate the interpretation.

\section{COST OF GETTING IT WRONG}

It is intuitive that clinicians seek to protect the interests of their patients, and some may wonder if a discussion of the quantification and interpretation of risk is relevant as opposed to acceptance and avoidance of risk based on published values. In the CALGB 9238 study, the largest in the series (with 422 patients), physicians were allowed to offer surgical treatment of patients with 'very high risk', defined as $\mathrm{FEV}_{1}<900 \mathrm{ml}$ and $\mathrm{VO}_{2}$ max of $<15 / \mathrm{ml} / \mathrm{kg} /$ min. Of the 68 patients in the 'very high risk' group, there was only one postoperative death within 30 days and a total of three in-hospital deaths. ${ }^{17}$ More importantly, on follow-up, the operated patients in the very high risk group had more than double the median survival compared with the non-operated patients ( 36.0 months vs 15.8 months, $p<0.001$ ), illustrating acceptable procedural mortality and morbidity with twice the median survival with case selection on parameters independent of $\mathrm{VO}_{2}$ max. Denying patients with 'prohibitive' values of $\mathrm{VO}_{2}$ max the opportunity to consider surgery as a management option may in fact be against their best interests. As the study was not randomised, it is important to bear in mind the invariable presence of selection bias, and the possibility that a better result was achieved by offering surgery to fitter patients with less co-morbidity. Our point is more to question the 'conventional' lower limit of safety and the results that can be achieved by further selection.

\section{THE FUTURE}

We acknowledge the consistent message that low levels of $\mathrm{VO}_{2}$ max are associated with increased complications from surgery. However, we believe current recommendations are flawed by small sample sizes, resulting in imprecise risk estimates. Moreover, the lack of numerical quantification leads to difficulties in defining the level of acceptable risk. Furthermore, the use of composite outcomes leads to a lack of agreement on the importance of the risks, and the incongruence limits the clinical applicability to inform patients on the decision to undergo surgery. We believe that management options should be discussed at a multidisciplinary level but decisions should be undertaken at patient level. This is because patients are heterogeneous, with individual perceptions on the value of benefit and risk. As the lower limits of safety remain imprecisely defined, patients with multidisciplinary team-defined 'prohibitive' levels of risk may not be offered the opportunity to consider surgery as an option and denied the possibility of increased life expectancy.

There may also be a degree of concern if postoperative quality of life may be a tradeoff for any increase in life expectancy in the high risk cohort; however, prospective studies indicated that patients traditionally considered at higher risk of lung resection had postoperative physical and emotional quality of life scores similar to those observed in younger and fitter patients. ${ }^{26}$

Before widespread use, further work needs to be performed to determine if cardiopulmonary exercise testing is an independent predictor of mortality (eg, above and beyond that of Thorascore), to relate the study to individual outcomes that would influence the decision to undergo surgery, to provide numerical quantification of risk with an estimate of uncertainty and to demonstrate validity in different cohorts.

\section{Competing interests None.}

Provenance and peer review Commissioned; externally peer reviewed.
Published Online First 13 July 2010

Thorax 2010;65:847-849.

doi:10.1136/thx.2009.133181

\section{REFERENCES}

1. Brunelli A, Charloux A, Bolliger CT, et al. ERS/ESTS clinical guidelines on fitness for radical therapy in lung cancer patients (surgery and chemo-radiotherapy). Eur Respir J 2009;34:17-41.

2. Colice GL, Shafazand S, Griffin JP, et al. Physiologic evaluation of the patient with lung cancer being considered for resectional surgery: ACCP evidencedbased clinical practice guidelines (2nd edition). Chest 2007;132(3 Suppl):161S-77S.

3. British Thoracic Society; Society of Cardiothoracic Surgeons of Great Britain and Ireland Working Party. BTS guidelines: guidelines on the selection of patients with lung cancer for surgery. Thorax 2001;56:89-108.

4. Bechard D, Wetstein L. Assessment of exercise oxygen consumption as preoperative criterion for lung resection. Ann Thorac Surg 1987;44:344-9.

5. Bolliger CT, Jordan P, Soler M, et al. Exercise capacity as a predictor of postoperative complications in lung resection candidates. Am J Respir Crit Care Med 1995;151:1472-80.

6. Boysen PG, Clark CA, Block AJ. Graded exercise testing and postthoracotomy complications. J Cardiothorac Anesth 1990;4:68-72.

7. Brunelli A, Belardinelli R, Refai M, et al. Peak oxygen consumption during cardiopulmonary exercise test improves risk stratification in candidates to major lung resection. Chest 2009;135:1260-7.

8. Brutsche MH, Spiliopoulos A, Bolliger CT, et al. Exercise capacity and extent of resection as predictors of surgical risk in lung cancer. Eur Respir $J$ 2000;15:828-32

9. Epstein SK, Faling LJ, Daly BD, et al. Predicting complications after pulmonary resection. Preoperative exercise testing vs a multifactorial cardiopulmonary risk index. Chest 1993;104:694-700.

10. Richter Larsen K, Svendsen UG, Milman N, et al. Exercise testing in the preoperative evaluation of patients with bronchogenic carcinoma. Eur Respir $J$ 1997:10:1559-65

11. Markos J, Mullan BP, Hillman DR, et al. Preoperative assessment as a predictor of mortality and morbidity after lung resection. Am Rev Respir Dis 1989;139:902-10.

12. Morice RC, Peters EJ, Ryan MB, et al. Exercise testing in the evaluation of patients at high risk for complications from lung resection. Chest 1992;101:356-61.

13. Smith TP, Kinasewitz GT, Tucker WY, et al. Exercise capacity as a predictor of post-thoracotomy morbidity. Am Rev Respir Dis 1984;129:730-4.

14. Torchio R, Gulotta C, Parvis M, et al. Gas exchange threshold as a predictor of severe postoperative complications after lung resection in mild-tomoderate chronic obstructive pulmonary disease. Monaldi Arch Chest Dis 1998;53:127-33.

15. Villani F, Busia A. Preoperative evaluation of patients submitted to pneumonectomy for lung carcinoma: role of exercise testing. Tumori 2004;90:405-9.

16. Wang J, Olak J, Ultmann RE, et al. Assessment of pulmonary complications after lung resection. Ann Thorac Surg 1999;67:1444-7.

17. Loewen GM, Watson D, Kohman L, et al. Preoperative exercise $\mathrm{V}_{2}$ measurement for lung resection candidates: results of Cancer and Leukemia Group B Protocol 9238. J Thorac Oncol 2007;2:619-25.

18. Bobbio A, Chetta A, Internullo E, et al. Exercise capacity assessment in patients undergoing lung resection. Eur $J$ Cardiothorac Surg 2009;35:419-22.

19. Page R, Keogh B. National Thoracic Surgery Activity and Outcomes Report. Oxford; Dendrite Clinical Systems Ltd, 2008. 
20. Schipper PH, Diggs BS, Ungerleider RM, et al. The influence of surgeon specialty on outcomes in general thoracic surgery: a national sample 1996 to 2005. Ann Thorac Surg 2009;88:1566-72;discussion 1572-63.

21. Benzo R, Kelley GA, Recchi L, et al. Complications of lung resection and exercise capacity: a meta-analysis. Respir Med 2007;101:1790-7.

22. Falcoz PE, Conti M, Brouchet L, et al. The Thoracic Surgery Scoring System (Thoracoscore): risk model for in-hospital death in 15,183 patients requiring thoracic surgery. J Thorac Cardiovasc Surg 2007; 133:325-32

23. Chamogeorgakis TP, Connery CP, Bhora F, et al. Thoracoscore predicts midterm mortality in patients undergoing thoracic surgery. J Thorac Cardiovasc Surg 2007;134:883-7.

24. Montori VM, Permanyer-Miralda G, FerreiraGonzalez I, et al. Validity of composite end points in clinical trials. Br Med J 2005;330:594-6.
25. Lim E, Brown A, Helmy A, et al. Composite outcomes in cardiovascular research: a survey of randomized trials. Ann Intern Med 2008;149:612-17.

26. Brunelli A, Socci L, Refai M, et al. Quality of life before and after major lung resection for lung cancer: a prospective follow-up analysis. Ann Thorac Surg 2007:84:410-16.

\title{
Pseudomonas aeruginosa infection in cystic fibrosis: prevent, eradicate or both?
}

\author{
Ernst Eber, Maximilian S Zach
}

Lung disease in cystic fibrosis (CF) starts early in life. Infection, inflammation, reduced lung function and abnormal chest CT findings are present in a significant proportion of infants with CF at a time when many of these children have no clinically apparent lung disease. ${ }^{1-6}$ Infection and inflammation are intimately linked early in the course of CF lung disease. ${ }^{2} 7-9$ The detection of airway inflammation in the absence of apparent infection led to the speculation that CF is associated with an intrinsic abnormality of immune regulation. ${ }^{10}$ However, more recent data argue against a primary CF-associated dysregulation of local immune function where inflammation might precede infection; they rather support the central role of bacterial pathogens in initiating and sustaining the neutrophil-mediated airway inflammation characteristic of CF lung disease, with a higher inflammatory burden in children infected with Pseudomonas aeruginosa (PA) than in those infected with organisms other than PA or uninfected. ${ }^{6} 1112$ Chronic respiratory infection is one of the main characteristics of CF and significantly contributes to morbidity and mortality. ${ }^{12}{ }^{13}$ In young children Staphylococcus aureus, Haemophilus influenzae and PA are the major lower airway pathogens. ${ }^{12} 13$ Chronic infection with PA is known to be associated with a worse prog-

Klinische Abteilung für Pulmonologie und Allergologie, Univ-Klinik für Kinder- und Jugendheilkunde, Medizinische Universität Graz, Graz, Austria

Correspondence to Dr Ernst Eber, Klinische Abteilung für Pulmonologie und Allergologie, Univ-Klinik für Kinderund Jugendheilkunde, Medizinische Universität Graz, Auenbruggerplatz 34, 8036 Graz, Austria;

ernst.eber@medunigraz.at nosis in children, adolescents and adults, and patients with mucoid PA strains do worse than those with non-mucoid strains. ${ }^{14-18}$ Thus, prevention or early detection and treatment of infection, in particular with PA, may prevent or delay irreversible lung damage from inflammation and consequently may improve prognosis. In order to prevent acquisition of PA, hygienic measures to decontaminate environmental reservoirs of this organism including medical equipment have been stressed, and CF centres have adopted meticulous microbiological surveillance and effective segregation policies to limit cross-infection between patients. Furthermore, several trials have been undertaken to assess the value of vaccination as a preventive strategy for PA infection but, with the variable outcomes of these trials, vaccines against PA cannot be recommended. ${ }^{19}$ At present, early treatment of initial infection-that is, an attempt to eradicate $\mathrm{PA}$-is the agreed standard. ${ }^{20}$ Literature on antibiotic prophylaxis of PA infection has so far remained scarce. ${ }^{21} 22$

In this issue of Thorax, TramperStranders et al report on a randomised controlled trial investigating the effectiveness of cycled antibiotic prophylaxis in children with CF (see page 915). ${ }^{23}$ Children between 0 and 18 years of age received 3 -monthly 3 -week treatments with oral ciprofloxacin and inhaled colistin or placebo for 3 years. The authors apparently chose this regimen because many CF centres across Europe have used the combination of oral ciprofloxacin and inhaled colistin as eradication therapy for $\mathrm{PA}$, going back to the first randomised controlled trial of an eradication regimen almost 20 years ago. $^{24}$ Patients were followed at 3-monthly intervals in between treatment cycles and respiratory specimens collected for microbiology studies were either oropharyngeal cough swabs or sputum samples (notably, the vast majority of patients did not produce sputum). In addition, serum samples were collected every 6 months for measurement of anti-Pseudomonas antibodies. Infection with PA (the primary end point) was defined as either two positive cultures taken $>1$ week apart or one positive culture and a 'severe pulmonary exacerbation'. Nineteen out of 65 patients (29\%) reached the primary end point (in only 4 of them was PA detected in sputum); information on how many strains were mucoid or non-mucoid is not given. Fourteen patients had positive antibody titres during the study period once or more often; 10 of them were culturepositive before they were serology-positive. This study once more shows that detection of infection in children with $\mathrm{CF}$ is difficult. In general, young children are not able to expectorate sputum, and oropharyngeal cultures do not reliably predict the presence of bacterial pathogens in the lower airways of young children with CF. ${ }^{13} 25$ Thus, cultures from bronchoalveolar lavage (BAL) fluid are usually taken as the gold standard for detection of infection in the lower airways. However, owing to sampling problems, even a negative BAL fluid culture or the absence of a particular organism on culture does not guarantee that the organism is not present in the lungs of the patient. ${ }^{26}$ For this reason, additional markers of infection are desirable and the use of specific serum antibodies against PA may be helpful in defining the status of PA infection. However, the majority of patients are antibody-negative at the time of first infection so specific antibodies against PA alone are not recommended to diagnose early infection. ${ }^{27}$ With oropharyngeal cough swabs being the prevailing respiratory specimens, one might speculate that at least some patients with positive upper airway cultures might have had negative 